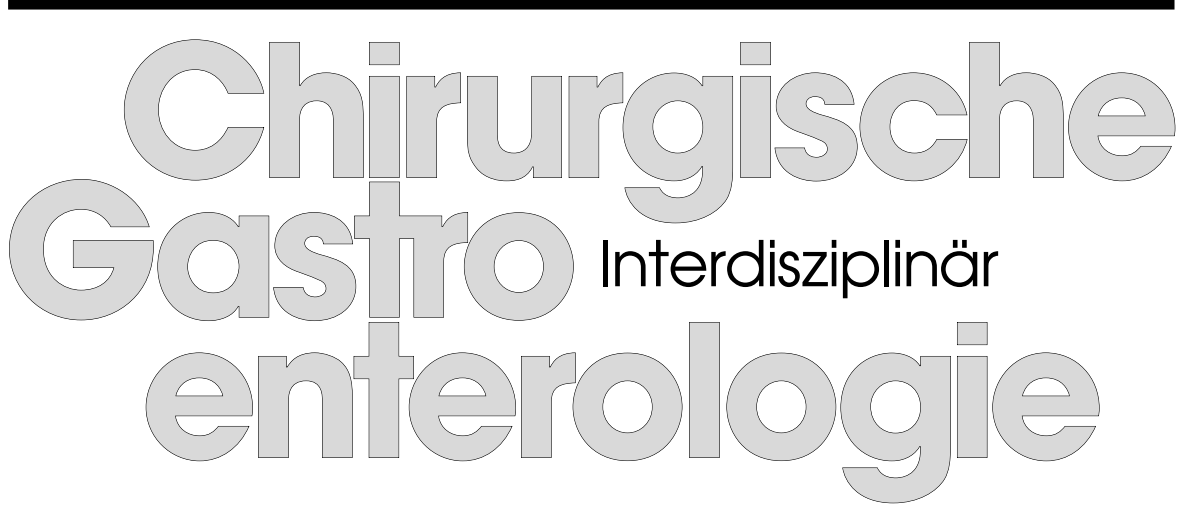

Hauptthema

B. Gloor, Bern

J. Mössner, Leipzig

Akutes Abdomen:

M.W. Büchler, Heidelberg Aktuelles aus internistischer
und chirurgischer Sicht (Hrsg.)

\author{
Eingeladene Beiträge \\ Interdisziplinäres Gespräch
}

Weitere Rubriken

Originalarbeit

Berufliche Weiterbildung

Innovationen

Tagungen und Kongresse 


\section{Band 18, Heft 3, September 2002}

Gegründet 1984 als «Chirurgische

Gastroenterologie mit interdisziplinären

Gesprächen» durch A. Akovbiantz, H. Denk,

K.-J. Paquet und C.E. Zöckler.

\section{Hauptschriftleitung}

R. Bittner, Stuttgart

\section{Schriftleitung Viszeralchirurgie}

E. Klar, Heidelberg

\section{Schriftleitung Gastroenterologie}

J. Mössner, Leipzig

\section{Assistenz Schriftleitung}

C.-G. Schmedt, Stuttgart

\section{Fachschriftleitung}

Viszeralchirurgie

J. R. Izbicki, Hamburg

H. Lippert, Magdeburg

H. Säuberli, Baden

R. Schiessel, Wien

Gastroenterologie

H. Bosseckert, Jena

W. G. Zoller, Stuttgart

Minimal invasive Chirurgie

H.-P. Bruch, Lübeck

I. Gastinger, Cottbus

Transplantation

C. E. Broelsch, Essen

J. Hauss, Leipzig

Berufspolitik

K.-J. Paquet, Hannover

B. Ulrich, Düsseldorf

Chirurgische Onkologie

M.W. Büchler, Heidelberg

K. Glaser, Wien

\section{Wissenschaftlicher Beirat}

\section{Viszeralchirurgie}

J. Lange, St. Gallen

H.B. Reith, Würzburg

H.-D. Saeger, Dresden

M. Schilling, Homburg/Saar

\section{Gastroenterologie}

T. Andus, Stuttgart

W. E. Fleig, Halle

M. Fried, Zürich

E. G. Hahn, Erlangen-Nürnberg

J. Schölmerich, Regensburg

Minimal invasive Chirurgie

F. Köckerling, Hannover

R. Schlumpf, Aarau

K. Schönleben, Ludwigshafen

G. Szinicz, Bregenz

W. Wayand, Linz

Berufspolitik

M. Betzler, Essen

K. Junghanns, Ludwigsburg

Chirurgische Onkologie

W. Hohenberger, Erlangen-Nürnberg

Onkologie

S. von Hegewisch-Becker, Hamburg

R. Porschen, Bremen

\section{Internationaler Beirat}

J. Gallinger, Moskau

J.M. Henderson, Cleveland, $\mathrm{OH}$

I. Ihse, Lund

C. W. Imrie, Glasgow

S. L. Jensen, Arhus

K. Maruyama, Tokyo

W. C. Meyers, Worcester, MA
Endoskopie

S. Liebe, Rostock

P. Malfertheiner, Magdeburg

J.F. Riemann, Ludwigshafen

Perioperatives Management

K.-W. Jauch, Regensburg

H. Wiedeck, Ulm

D. H. Wittmann, Venice, FL

K. Hell, Basel

Radiologie

M. Langer, Freiburg i. Br.

Pathologie

P. Hermanek, Erlangen-Nürnberg

C. Wittekind, Leipzig

Klinische Molekularbiologie

H. Friess, Heidelberg

Weiterbildung

J. Jähne, Hannover

Innovationen, Aktuelle Trends,

Kongressberichte

J. Schmidt, Heidelberg

J. Mössner, Leipzig

W. G. Zoller, Stuttgart

Buchbesprechungen

K.-J. Paquet, Hannover

E. Moreno-Gonzáles, Madrid

F.M. Penninckx, Leuven

A. Peracchia, Mailand

L.F. Rikkers, Madison, WI

J. Rodés, Barcelona

A.L. Warshaw, Boston, MA

Y. Yamamoto, Kyoto

S. Karger $\cdot$ Medical and Scientific Publishers $\cdot$ Basel $\cdot$ Freiburg $\cdot$ Paris $\cdot$ London $\cdot$ New York $\cdot$ New Delhi $\cdot$ Bangkok $\cdot$ Singapore $\cdot$ Tokyo $\cdot$ Sydney

Die Zeitschrift erscheint vierteljährlich; pro Jahr erscheint 1 Band zu je 4 Heften. Bezugspreis für Jahrgang 18, 2002: EUR 144,- / SFr 202,-- einschließlich MwSt., zuzüglich Postgebühren. Der Abonnementpreis ist im Voraus zahlbar. Das Abonnement der Zeitschrift läuft weiter, wenn es nicht spätestens 4 Wochen vor Abschluss eines Bandes abbestellt wird.

Abonnementbestellungen können bei jeder Buchhandlung oder direkt beim Verlag aufgegeben werden:

Deutschland:

S. Karger $\mathrm{GmbH}$

Lörracher Str. 16a

D-79115 Freiburg

Tel. +49761452070

Fax +497614520714

E-mail Information@Karger.de
Übrige Länder:

S. Karger AG

Allschwilerstr. 10

CH-4009 Basel

Tel. +41613061111

Fax +41613061234

E-mail Karger@Karger.ch

\section{Anzeigen: S. Karger}

Verlag für Medizin und Naturwissenschaften $\mathrm{GmbH}$ Lörracher Str.16a, D-79115 Freiburg, Tel. +49 761452070. Gültig ist die Preisliste Nr. 9 vom 1. Januar 2002.

Für den Inhalt ausserhalb des redaktionellen Teiles (insbesondere Anzeigen, Industrieinformationen, Pressezitate und Kongressinformationen) übernehmen Schriftleitung, Beirat und Verlag keine Gewähr.

Eine Markenbezeichnung kann warenzeichenrechtlich geschützt sein, auch wenn bei ihrer Verwendung in dieser Zeitschrift das Zeichen ${ }^{\circledR}$ oder ein anderer Hinweis auf etwa bestehende Schutzrechte fehlen sollte. Für Satzfehler, insbesondere bei Dosierungsangaben, wird keine Gewähr übernommen.

Die Zeitschrift sowie alle in ihr enthaltenen einzelnen Beiträge und Abbildungen sind urheberrechtlich geschützt. Jede Verwertung, die nicht ausdrücklich vom Urheberrechtsgesetz zu- gelassen ist, bedarf der vorherigen Zustimmung des Verlags. Das gilt insbesondere für Vervielfältigungen, Bearbeitungen, Übersetzungen, Mikroverfilmungen und die Einspeicherung und Verarbeitung in elektronischen Systemen.

(c) Copyright 2002 by S. Karger

Verlag für Medizin und Naturwissenschaften $\mathrm{GmbH}$ Lörracher Str. 16a, D-79115 Freiburg

Verlagsleitung und presserechtlich verantwortlich: Sibylle Hopf

Redaktionsassistenz: Dr. Hannelore Tenckhof Anzeigenleitung: Susanne Meister Produktionsleitung: Georg Brunner

Gesamtherstellung: KONKORDIA GmbH, Büh Das Medienunternehmen

ISBN 3-8055-7436-3

\section{KARGER}

Fax +497614520714 E-mail Information@Karger.de www.karger.com

\section{(C) 2002 S. Karger GmbH, Freiburg}

Bibliographische Dienste: EMBASE / Excerpta Medica Reference Update 
Band 18, Heft 3, September 2002

Laudatio

193 Statt einer Laudatio:

Zum 65. Geburtstag von Karl-Joseph Paquet

Zöckler, C.E. (Bad Oeynhausen)

195 Editorial

Gloor, B. (Bern); Mössner, J. (Leipzig); Büchler, M.W. (Heidelberg)

Hauptthema

196 Das akute Abdomen - eine Übersicht

Leibl, B.L.; Schmedt, C.G.; Bittner, R. (Stuttgart)

202 Akutes Abdomen und Peritonitis: Pathophysiologie und Therapiekonzepte

Heinicke, J.-M.; Ly, Q.; Seiler, C.A.; Candinas, D.; Gloor B. (Bern)

208 Cholelithiasis

Heller, J; Sauerbruch, T. (Bonn)

216 Akute Pankreatitis - intensivmedizinische oder chirurgische Therapie?

Strobel, O.; Uhl, W.; Müller, C.A. (Heidelberg); Gloor, B. (Bern);

Werner, J.; Büchler, M.W. (Heidelberg)

\section{Akute Appendizitis}

Koch, A.; Marusch, F. (Cottbus); Wolff, S. (Magdeburg); Gastinger, I. (Cottbus); Lippert, H. (Magdeburg)

230 Notfälle beim Morbus Crohn: Wann soll operiert werden?

Schmidt, J.; Heuschen, U.; Eisold, S.; Kienle, P.; Klar, E. (Heidelberg)

238 Notfallindikationen und Operationsverfahren bei toxischer Colitis ulcerosa

Heuschen, U.; Heuschen, G.; Klar, E. (Heidelberg)

244 Ileus als Ursache des akuten Abdomens Bruch, H.-P.; Schwander, O.; Markert, U. (Lübeck)

252 Vaskuläre Ursachen des akuten Abdomens: Haben wir Fortschritte gemacht? Kniemeyer, H.; Edelmann, M. (Essen); Reber, P.U. (Bern)
Vol. 18, Issue 3, September 2002

Laudation

193 Instead of a Laudation:

To Karl-Joseph Paquet's 65th Birthday

Zöckler, C.E. (Bad Oeynhausen)

195 Editorial

Gloor, B. (Bern); Mössner, J. (Leipzig); Büchler, M.W. (Heidelberg)

Main Topic

196 Acute Abdomen - an Overview

Leibl, B.L.; Schmedt, C.G.; Bittner, R. (Stuttgart)

202 Acute Abdominal Pain and Peritonitis: Pathophysiology and Therapeutic Concepts

Heinicke, J.-M.; Ly, Q.; Seiler, C.A.; Candinas, D.; Gloor B. (Bern)

208 Cholelithiasis

Heller, J.; Sauerbruch, T. (Bonn)

216 Acute Pancreatitis - Conservative Intensive Care or Surgical Therapy?

Strobel, O.; Uhl, W.; Müller, C.A. (Heidelberg); Gloor, B. (Bern); Werner, J.; Büchler, M.W. (Heidelberg)

224 Acute Appendicitis Koch, A.; Marusch, F. (Cottbus); Wolff, S. (Magdeburg); Gastinger, I. (Cottbus); Lippert, H. (Magdeburg)

230 Emergency Cases in Crohn's Disease: When to Operate? Schmidt, J.; Heuschen, U.; Eisold, S.; Kienle, P.; Klar, E. (Heidelberg)

238 Emergency Indications and Surgical Therapies in Patients with Toxic Colitis ulcerosa Heuschen, U.; Heuschen, G.; Klar, E. (Heidelberg)

244 Intestinal Obstruction as Cause of Acute Abdomen Bruch, H.-P.; Schwander, O.; Markert, U. (Lübeck)

252 Vascular Causes of Acute Abdomen:

Is There a Progress?

Kniemeyer, H.; Edelmann, M. (Essen); Reber, P.U. (Bern) 


\section{Band 18, Heft 3, September 2002}

260 Das akute Abdomen aus intensivmedizinischer Sicht Bartels, H. (München)

266 Akutes Abdomen: Wann sollte nicht operiert werden? Mössner, J. (Leipzig)

Interdisziplinäres Gespräch

272 Akutes Abdomen - eine interdisziplinäre

Herausforderung

Teichmann, W. (Hamburg) (Gesprächsleiter)

Originalarbeit

282 Epidemiologie der Analinkontinenz

Roche, B.; Chautems, R.; Rakotoarimanana, R.; Berclaz, O.;

Marti†, M.-C. (Genf)

Berufliche Weiterbildung

286 Aktueller Stand der zukünftigen Weiterbildungsstruktur fuir das neu geschaffene Gebiet Chirurgie Witte, J. (Berlin)

289 Innovationen

291 Tagungen und Kongresse

190 Impressum

U3 Hinweise für Autoren (3. Umschlagseite)
Vol. 18, Issue 3, September 2002

260 Acute Abdomen for Intensive Care Medicine Purposes Bartels, H. (München)

266 Acute Abdomen: When Not to Operate? Mössner, J. (Leipzig)

Interdisciplinary Discussion

272 Acute Abdomen - an Interdisciplinary Challenge Teichmann, W. (Hamburg) (Discussion Leader)

Original Article

282 Epidemiology of Anal Incontinence Roche, B.; Chautems, R.; Rakotoarimanana, R.; Berclaz, O.; Marti†, M.-C. (Genf)

Professional Education

286 Future Organization of Further Education in the Recently Created Field of Surgery - State of the Art Witte, J. (Berlin)

289 Innovations

291 Meetings and Conferences

190 Imprint

U3 Guidelines for Authors (Inside back cover)

\section{KARGER}

(c) 2002 S. Karger GmbH, Freiburg

Fax +497614520714 